\title{
Attention and Communication: Decision Scenarios for Teleoperating Robots
}

\author{
Jeffrey V. Nickerson \\ Stevens Institute of Technology \\ jnickerson@stevens.edu
}

\author{
Steven S. Skiena \\ State University of New York at Stony Brook \\ skiena@cs.sunysb.edu
}

\begin{abstract}
The economics of robot manufacturing is driving us toward situations in which a single human operator will be expected to split attention across multiple semiautonomous vehicles, and remotely intercede if necessary. We present an analysis of such situations, with the goal of creating decision aids. Toward this end, the concept of special regions is introduced. In one set of situations special regions designate areas that are dangerous, and require teleoperation. We show how to move through single route and multi-route situations, and prove the later problem NP-Complete. In another set of situations, special regions can be used to represent areas outside direct radio contact. We present a way to minimize communication distance and plan for interventions. We relate our findings to concepts of neglect time, interaction time, and fan-out. We discuss a measure of effective fanout for transportation tasks, and present simulation results. The work has potential impact to those engaged in emergency response and search and rescue.
\end{abstract}

\section{Introduction}

We can now build robotic vehicles that are semiautonomous. At present, these robots can, in certain well understood conditions, move without harming people or property. But, in other conditions, which are either complex or unexpected, they require intervention; an intervention may be a simple corrective command or fullscale teleoperation by a human. This intervention might be required to get the robot out of a stuck situation, or to prevent a malfunctioning robot from harming people or property.

These machines are capable of running at different degrees of autonomy, and there is an expanding literature which discusses how levels of autonomy might be changed dynamically - in other words, how control might be ceded to robot and then ceded back to a human, depending on the situation encountered at a particular instant in time [1-8]. The research area of remote control of semi-autonomous land vehicles for search and rescue has received recent attention [9-15].

One striking theme of the literature involves minimizing human cognitive load. In effect, a machine which detects that its human operator is overloaded may increase its level of autonomy. For example, in order to avoid collision, a machine might take over the controls from a human pilot $[16,17]$.

In such a condition, ceding control may make sense machines may do better at making the decision to avoid a collision, especially if the other vehicle is also controlled by a machine (e.g. [18]). But on consideration, the reader may find there is a certain irony - as situations become more complex, one theoretically might want a decision to be made by a human - but instead, in order to minimize load on the human, the machine will end up taking control and making the decision. We may be ceding control at the exact instant that human control is needed.

Let us consider a situation in which we might want robots to cede control to a teleoperator. Imagine semiautonomous automobiles negotiating a city with many traffic circles. The algorithms of the vehicle may work very well in highway driving, but may not be able to handle traffic circle merges, in which not only the pattern of the traffic, but the subtlety of head movement and eye contact may help drivers make decision about when to merge. One might want control to cede back to a teleoperator for those portions of a journey.

Now consider a second constraint. If we build remotely controllable vehicles at great expense, and need to assign one person to operate each vehicle, then we have achieved little if any financial advantage over a manually operated vehicle. So, in many discussions of the control of semiautonomous vehicles, operators are expected to handle multiple vehicles (e.g. [13]); the number to be controlled is often mentioned as about four.

Yet we don't really know if this is possible, or whether a higher or lower ratio of human to machine would be

Copyright 2005 IEEE. Proceedings of the Hawai'i International Conference on System Sciences, January 3 - 6, 2005, 
possible. We sense that the ratio will be dependent on situation.

Scholtz, Antonishek, and Young [19] articulate a way to evaluate situation awareness - and observe that operators need a way of anticipating when events will occur so that they can judge what interactions will be necessary. In a separate study, they show that the average intervention time in remote driving was 161 seconds [13]. While they observe that better interfaces might reduce this time, and while we can't necessarily generalize from one study, it is worth considering how truly long this is. It leads us to wonder if we can reasonably expect an operator to shift from monitoring to teleoperation while continuing to monitor the other vehicles.

Olsen and Wood, in presenting a theory of fan-out, the number of robots an individual can control, show how at some point the addition of new robots will add little to the performance of search tasks [20]. The authors describe activity time as the time a robot can act effectively before it needs a new command. They distinguish this from neglect time, the time during which a robot can be trusted to operate without supervision; this concept is also discussed in [21, 22]. Olsen and Wood point out a robot may be capable of acting on its own, but we may still not want to neglect it. The authors also describe interaction time, which includes the time a human spends monitoring or operating a robot. They also include in this measure the time it takes to switch attention between two robots, as well as the offline planning time to solve a problem. While their work has focused on search tasks, this paper focuses on transportation tasks, with all their related constraints associated with sequencing and safety.

We want to build decision aids that would help in both the planning and control of missions involving remote vehicles in the domain of emergency response. In such situations, planners want to make sure the robot doesn't harm others. And often the planners are faced with situations in which surrounding communication infrastructure fails. We are specifically interested in how we can reason about the control of multiple robots in such circumstances, and, if we can, what kind of human:robot ratio is really achievable. First we look at issues of teleoperation. Then we look at communication. Next, we analyze attention in more detail, and finally we discuss the calculation of fan-out.

\section{Moving through dangerous regions}

Scenario 1: An operator is teleoperating 3 semiautonomous land vehicles that will take the same route through an urban environment. The vehicles can control their speed, but must keep moving. The vehicles are known to have problems negotiating traffic circles. How should the 3 vehicles be spaced?

Let us assume that that there are 3 traffic circles. These circles are special regions we designate on a map; in this situation, these regions are difficult for the robotic vehicles to traverse, and are therefore dangerous - to the robots, and to the surrounding traffic.

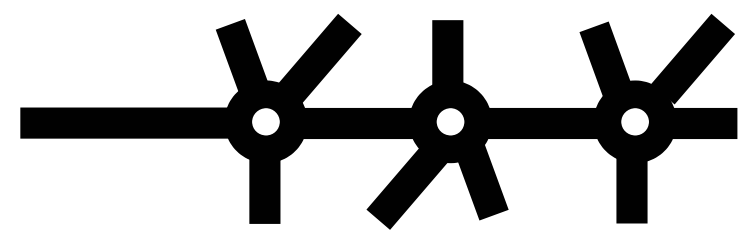

Figure 1. Rotaries as special regions that might require teleoperation

In the terminology of Olsen and Wood [20], we cannot neglect the robots in the traffic circle, and our full attention will be required to interact with a robot while it is in the circle. So our interaction time corresponds roughly to the length of time it takes to traverse the circles, and otherwise we neglect the robot. In actuality, we would need to ramp up our attention a little before entering the circle and taking control, as in figure 2, in order to build up our situational awareness.

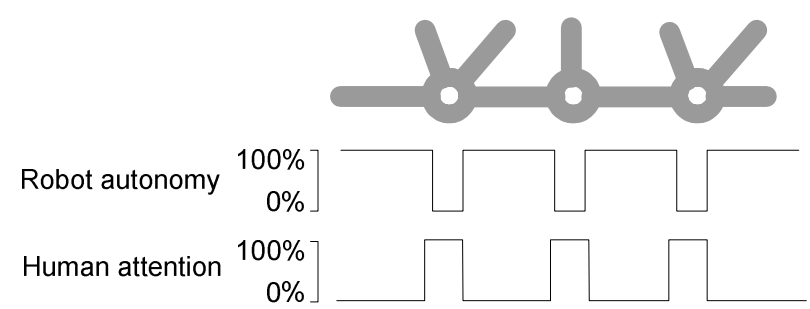

Figure 2. When the car enters each special region, autonomy drops in the robot as an operator devotes full attention to the task.

If we choose, we can space the cars apart so that the second car doesn't enter the first circle until the first car has cleared all three circles. But then we would gain no leverage.

How close can we make the cars? We cannot make them any closer than the length of the longest traffic circle - for then we would have two cars going through a dangerous region at the same time. We are assuming that can't do this - that an operator is only capable of taking full control of one car at a time. And if we increase the spacing, without prior planning, we can easily run into situations in which two cars are entering two traffic circles both at the same time. 
Let's look at how the situation unfolds. In figure 1, the rotaries represent special regions that will require teleoperation.

We can abstract this situation of rotaries by taking the intended path through the rotaries, linearizing it, quantizing it and showing the special regions distinct from the rest of the road.

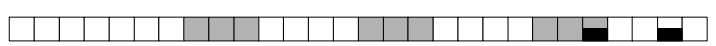

a)

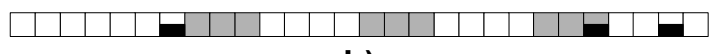

b)

Figure 3. a) Abstracting the special regions and placing two vehicles. b) Adding a third vehicle

With two cars, we can space them three lengths apart, and never have more than one in the special regions; in figure 3a, we show cars in black, and one can imagine the cars traversing the entire route from left to right - it is clear only one at a time will be in the rotary. But to add a third car is hard - the optimal solution is to add the car behind the last rotary, as shown in figure $3 \mathrm{~b}$.

We can visually demonstrate this - in figure 4 , for each closer combination of three cars, we show a situation in which two cars are in the rotaries.

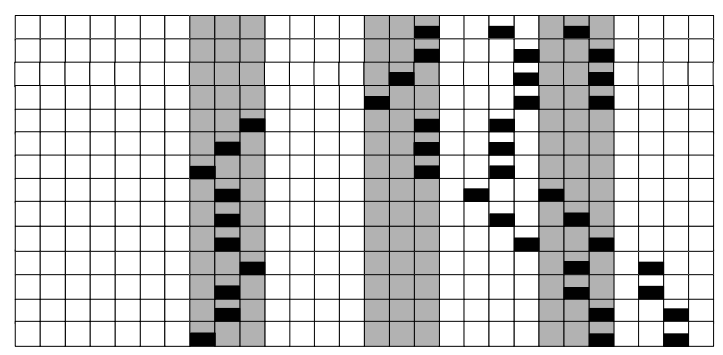

Figure 4. Showing alternate positions of the vehicles - in all positions, two cars are shown in special regions.

More formally, we can define the problem in the following way; given a path $P$, of length $D$, a set of regions, $R_{1} . . R_{n}$ with a function $L: R \rightarrow \mathbb{Z}^{*} \times \mathbb{Z}^{*}$ defining intervals on $P$, and a set of vehicles $V_{1} . V_{n}$ find a vehicle spacing vector $S_{1} . S_{n-1}$ so that, for all possible positions along $P$, there is at most 1 vehicle in any region interval. Why at most 1 ? Because when each vehicle is in a special region, an interaction with a human will be taking place, as in figure X. Since a human's attention will be consumed $100 \%$ during those times, and we are limiting operations to single person, we cannot permit more than vehicle in a special region at a time.

Figure 4 suggests an algorithm - find the closest arrangement between the first two cars, and then for each subsequent car, try all positions, and slide the cars until they fit. However, this greedy algorithm will not always provide the optimal solution. Figure 5c shows a shorter overall solution, than figure $5 \mathrm{~b}$, even with a greater distance between the first 2 cars. Figure $5 \mathrm{~d}$ suggests a way of finding the optimal solution - consider multiple copies of the route across all possible shifts, under the constraint that there can be only one gray square along a vertical column. Within the resulting set, find the configuration with the shortest overall length.

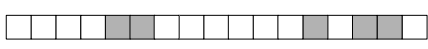

a)

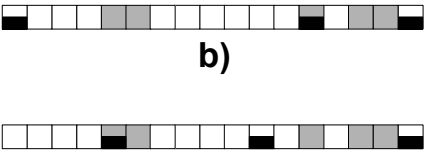

c)

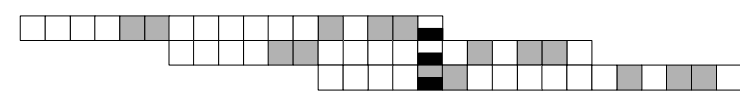

d)

Figure 5. a) The problem situation b) One solution c) A shorter solution d) Another way of representing $\mathrm{c}$.

Scenario 2: An operator is teleoperating 3 semiautonomous land vehicles that will take different routes through an urban environment. The vehicles can control their speed, but must keep moving. The vehicles are known to have problems negotiating traffic circles. How should the 3 vehicles be spaced?

This problem can be solved in a similar way. For each route, we create a distinct route vector, and then we shift the vectors until we find a situation of no overlap. Figure 6 shows an example.

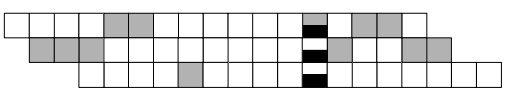

Figure 6. A solution in which the vehicles all take different paths.

Do we have to look at all combinations? Unfortunately yes, as the problem can be reduced from 3-Partition. 3Partition is NP-complete in the strong sense [23]. Let us 
consider a set $A$ of $3 m$ integers and a target integer B. We want to find if A can be partitioned into $m$ subsets, each of 3 elements, such that each subset adds up to exactly $B$.

The reduction is as follows. We will contruct $m$ bounding strings of the form

$$
1^{B+1} 0^{B} 1^{B+1}
$$

We will also construct one element string for each of the $3 m$ elements of $A$, namely $A_{i}$ written in unary, i.e.

$1^{A_{i}}$, for each $1 \leq i \leq m$.

Clearly no two bounding strings can overlap, because the runs of 1 are too long. The only way a solution of length $(3 B+2) m$ is possible is if each of the element strings pack with the gap in the bounding strings, which is only possible if we have a three-partition.

So, in order to find the optimal solution, we will be forced to consider many possibilities. For our examples, with probable maximum robot fan-out of 5 , we can still exhaustively search for solutions in the RouteSteps ${ }^{5}$ space that corresponds to shifting of routes in figure 6. But for problems with higher fan-out, a heuristic will be needed to approximate the optimal solutions.

There are also implications for the operator. Negotiating 3 different paths at the same time will increase context switching and complicate acquiring situational awareness. But it may also give the decision aid some leeway in planning - in an urban environment, particular paths might be picked out of the many possible in order to minimize potential overlapping of dangerous regions at the same time. Multiple path options will generally result in shorter overall solutions; later in the paper we will discuss why this is.

Now we vary one of the assumptions of scenario 1 :

Scenario 3: An operator is teleoperating 3 semiautonomous land vehicles that will take the same route through an urban environment. The vehicles are at the mercy of surrounding traffic. The vehicles are known to have problems negotiating traffic circles. How should the 3 vehicles be spaced?

In this scenario, it is possible that cars in front of the leading car will slow it down, and trucks in back of a following car will speed it up, to the point where the distance between the two cars are compressed. From the analysis before, it is clear that in such circumstances, the following car may be forced to enter a dangerous zone before the leading car has left. This may not be tenable, and so in such a situation, the correct algorithm is to send the cars one at a time through the city. How about if we vary the requirement differently?
Scenario 4: An operator is teleoperating 3 semiautonomous land vehicles that will take the same route through an urban environment. The vehicles can pull over and stop at will. The vehicles are known to have problems negotiating traffic circles. How should the 3 vehicles be spaced?

In this situation, it is possible to program the robots more dynamically - each robot may be informed when the operator is attending to another robot in a dangerous region, and will therefore pull over and stop before entering a dangerous zone itself.

There are also variations in which the traffic constraints disappear.

Scenario 5: An operator is teleoperating many rescue robots streaming into the rubble of an earthquake looking for survivors. How should the vehicles be spaced?

Whereas in traffic, a robot misperforming may hurt other people, in the scenario above, the robot has less chance of injuring someone else, and the robot may be seen as expendable. The robot could, however, block the path of following robots. So in such a situation, it may make sense to disperse the robots and let them search in parallel, without following each other. The operator may focus attention on the robots which make the most progress, and ignore robots that get stuck. This scenario is close to the search situations analyzed in [20].

\section{Communication}

Let us now imagine that the regions we discussed before are not regions that are difficult for the autonomous vehicle to traverse, but instead are regions in which we anticipate we will lose communication, such as tunnels.

Scenario 6: An operator is teleoperating 3 semiautonomous land vehicles that will take the same route through an urban environment. The vehicles are going to go through a series of tunnels, and communication will be lost as they pass through. How should the 3 vehicles be spaced?

In the first situation we couldn't have two vehicles in the special region because one would probably crash. In this situation, we are assuming that the vehicles will perform well in an autonomous mode while in the tunnels. 
But, if the journey involves uncertainty, we may want to characterize the extent to which we are in contact with the vehicles - a plan which sends all vehicles into a tunnel at once means we must wait until they reappear on the other side. What if there was an accident? Or we want to get some information from a vehicle near the emergency? For entirely different reasons than the first scenario, we may want to make sure only one vehicle at a time is in a tunnel.

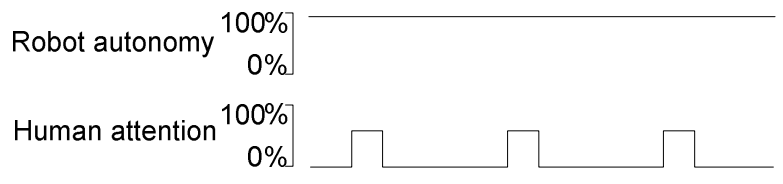

a)

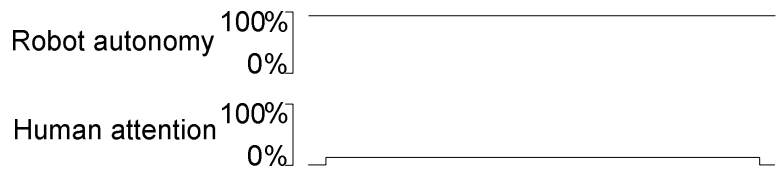

b)

Figure 7. In a), an operator periodically polls a robot, devoting half-attention for a small amount of time. In b), the operator devotes a small amount of continuous attention to a robot.

To be clear, in figure 2 we showed a model in which attention was only directed toward a robot when it entered a complex situation where the robot gave up autonomy. But also possible is a model such as that in $7 \mathrm{a}$, in which the robot preserves autonomy, and a human periodically polls the robot to see if it is OK. Such polling might not require full attention. Also possible is a model in which a small amount of continuous attention is directed toward a robot, as in $7 \mathrm{~b}$. This might occur if, for example, a supervisor were to monitor an overview map showing the locations of many robots as they move toward a destination.

Another way of putting is that we are assuming that the activity time of the robot includes the entire trip; the robot is strongly autonomous, and there is nothing in the geography of the route that calls for teleoperation. But we don't want to neglect the robot; we want to have the continuous ability to monitor the robot. We don't want our ability to monitor to be constrained due to a broken communication link. In other words, as a prerequisite to close monitoring, we need connectivity to the remote robots.

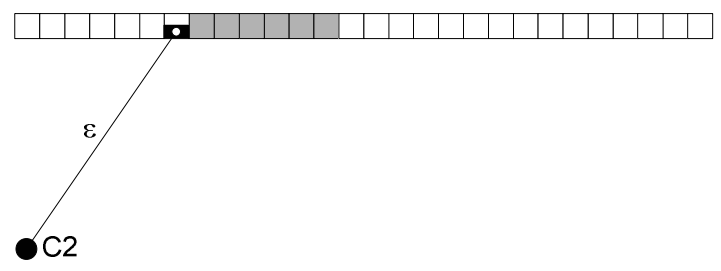

a)

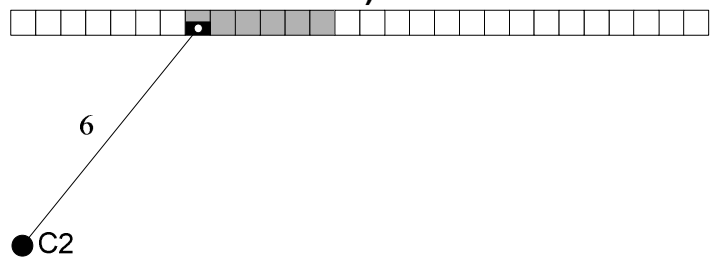

b)

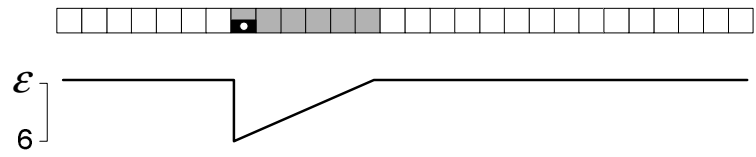

c)

Figure 8. The vehicle entering a tunnel; in a) communication is instant; in b) communication will be lost for 6 seconds; c) shows the ramp down of time to communicate.

But there are situations in which this might be difficult. In figure 8 , a vehicle is about to enter a tunnel, which will occlude radio communication. A supervisor (C2) is communicating to the vehicle. How can we more formally describe this? In a concurrent paper, we describe a metric we call communication distance, which is essentially a view of latency which is expanded to include the travel time it may take to establish communication [24]. In figure $8 \mathrm{a}$, the communication is through radio, and is practically instant, and so is labeled with epsilon.

In figure $8 \mathrm{~b}$, the car has moved into the tunnel, and the connection is broken. But if we know that the vehicle has entered the tunnel, then we know when we can communicate again - in this case in six intervals, and so we label the edge with a 6 . Figure $8 \mathrm{c}$ shows how the distance counts down from 6 back to epsilon as the car emerges from the tunnel.

Why are we stressing this? Because it is likely in emergency situations that communication will be lost and it may even be predictable where the communication is lost. How might we be able to predict? We would need maps of connectivity, as explained in [25].

If we analyze a situation using such maps, and find that we will lose communication along a route, then we may respond with two very different strategies. In the first, we treat the lack of communication as just another piece in 
the puzzle, and schedule our neglect times to correspond to times when the robot will be in a communication dead zone. So we would use a scheme such as that in $7 \mathrm{a}$. In the second, we decide we can't tolerate a loss of communication, that we need a scheme such as in $7 \mathrm{~b}$, so we configure the car deployments in such a way that the cars themselves bridge communication gaps.

Scenario 7: An operator is teleoperating 3 semiautonomous land vehicles that will take the same route through an urban environment. The vehicles are going to go through a series of tunnels, and direct communication to the dispatcher will be lost as they pass through. The vehicles have their own ad hoc network with which they can talk to each other within a limited radius. How should the 3 vehicles be spaced?

We may have a particular form of communication -say satellite - to our vehicles, and the vehicles themselves may communicate with each other over an ad hoc network.

In such a condition it might make sense to stagger the travel through the tunnel so that there is always at least one vehicle either about to enter or already leaving the tunnel - that way, connectivity is assured.

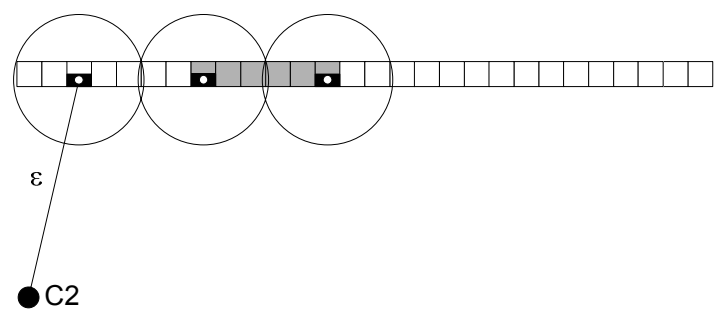

a)

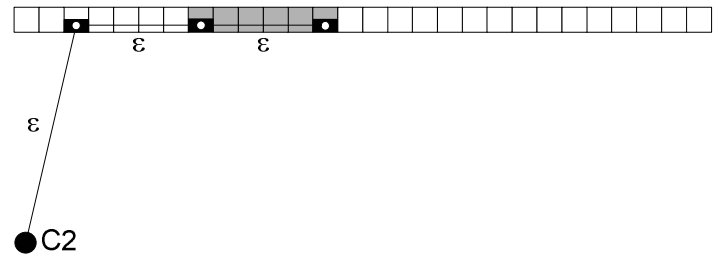

b)

Figure 9. a) Communicating to all vehicles through the ad hoc network. b) Communicating with the vehicle outside the tunnel.
Figure 9a shows two vehicles in the tunnel; if we establish communication to the vehicle outside the tunnel, then through the ad hoc network we can talk to all of them.

Figure $9 \mathrm{~b}$ shows this graphically - the communication to the vehicle outside is instant, and, assuming some form of network bridging, the ad hoc network allows the communication to all the vehicles to be instant.

So in this situation, we should not be concerned about having two cars in the tunnel - we should design the route so there is always one vehicle in communication with dispatch, and all other vehicles are within the ad hoc network's range.

Scenario 8: An operator is teleoperating as in the previous situation. One vehicle doesn't come back out of the tunnel at the expected time, and the leading car has kept on going. What should be done?

The effective communication distance to the stuck vehicle appears to be infinite - it didn't come out when it was supposed to, and there is no direct way to communicate with it from dispatch. But we can possibly use another vehicle - the vehicle ahead can turn around and come back, as in figure 10a.

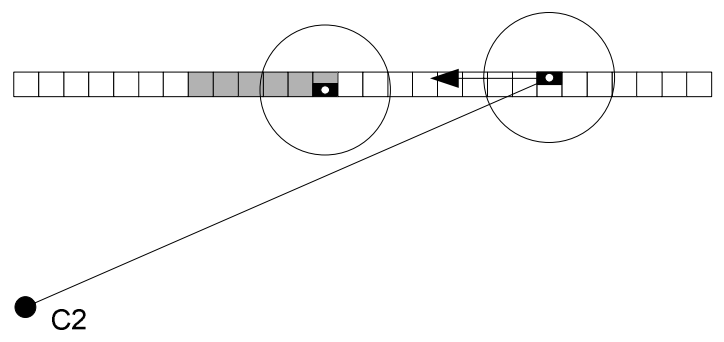

a)

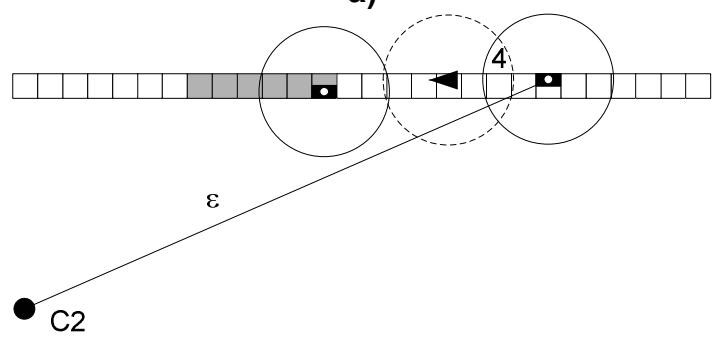

b)

Figure 10. a) Reestablishing communication with a stuck vehicle. b) The communication distance to the stuck vehicle is the time to move back into ad hoc network contact. 
So what is the communication distance to the stuck vehicle? It is the amount of time it will take the lead vehicle to move back into its radio range, if its radio is still working, as in figure $10 \mathrm{~b}$, or to physically intervene, if the radio is not working.

Why do we mention intervention? Sometimes it may make sense to intercept a malfunctioning vehicle in order to fix it or keep it from harming others. Olsen and Wood explicitly leave safety issues out of their consideration [20]; we propose communication distance as a way of reasoning about interventions when a malfunctioning robot cannot be neglected.

When teleoperation is called for, not only the presence of communication, but the amount of bandwidth may be important. For example, in order to negotiate a traffic circle, an operator may require a high throughput link to see a real-time video feed, and require the latency of the link to be low so that avoidance and braking maneuvers don't lag. Ways these more complex requirements can change route planning have been discussed in [24].

\section{Attention}

If we wish to monitor robots, then, as prerequisite resource, we need a working communication infrastructure, as we have just discussed. But even if we have a working infrastructure, the limitations of our own attention may limit our communication. As we become busy with one robot, our neglect times to other robots may be increasing.

What happens when an operator monitoring several vehicles switches attention to teleoperate a specific one?

Scenario 9: An operator is monitoring three vehicles, and one is about to go into a dangerous zone. How will communication with the other vehicles be affected?

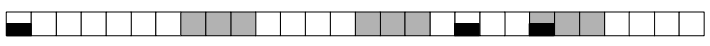

Figure 11. The situation of scenario 9.

It is clear from figure 11 that the first car is in a dangerous region - when it leaves, the second car will enter, and then the third. Assuming that this period of monitoring will require total concentration, the time to the next interactions are shown in figure 12.

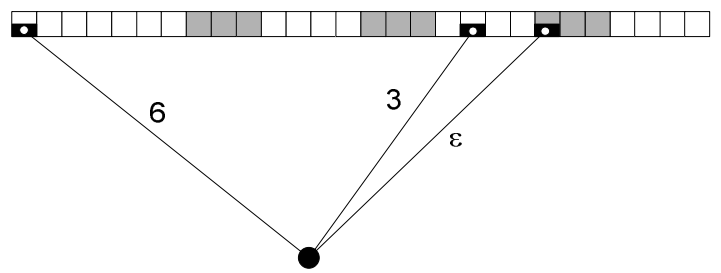

Figure 12. Monitoring three vehicles. The rightmost vehicle is currently in the focus of attention - the other vehicles will be attended to later.

At the instant at which control needs to be taken, the distance to the other cars increases - as the other cars will not be polled until the teleoperation is complete. Recall that the first scenario looked at situations where we know when the car will be teleoperated, and therefore could plan a route that effectively minimized the communication distance to the overall set of vehicles, by insuring that only one at a time would be in a critical region. But such requirements for teleoperation might also occur as a result of an emergency.

Before, we cited a test in which teleoperation times were on average 161 seconds [13]. Figure 12 indicates that we might end up with cascading times - while an operator is in one intervention, the effective communication time to the last car will be multiplied - for a situation with 4 cars, using 161 seconds per car, the last car would remain unmonitored for over 8 minutes.

In some situations, there may be hours of uneventful monitoring followed by minutes in which more than one person is required to monitor and teleooperate several vehicles.

Where is there an analogy to this? In call center environments, the overall call volume is understood statistically, but certain kinds of emergencies can increase call volume. Once an operator is engaged in a long call, the queues build - the distances increase. Calls are redistributed to other operators. But even this may not work, as a local problem will flood a local call center. More sophisticated centers use a backup call center in a different time zone, on the assumption that they will be less loaded, and can handle an increase in traffic.

The distinction often made between robotic supervision and operation gets at this problem. A supervisor might be watching what is happening - monitoring with partial attention, as in figure 7 - and when something seems to require intervention, might delegate the problem to the next available operator, who would be expected to spend full attention on the intervention.

In order to achieve high fan-out, instead of assigning a single operator to 4 remote vehicles, one might assign a single supervisor to many more vehicles for monitoring. When a vehicle goes into a dangerous region, the supervisor assigns an operator. 
We want to be able to handle preassigned teleoperation areas - as with our rotary examples. Also, we may want to be able to poll the robots - to periodically step into the robots environment, on the assumption that a human will be able to anticipate upcoming problems with greater skill than a robot. Both of these can be planned for.

But we also need to consider contingencies. In the case of a robot that can signal a problem, we want to be able to respond within a certain amount of time to a response for help ("I see a breakdown up ahead - requesting teleoperation"). Techniques for generating interrupts discussed in $[26,27]$ might be applicable to the operation of semi-autonomous vehicles, and relate to ideas for more cooperative interfaces [28].

How does this relate to neglect time? Neglect time is seen as a random variable related to time off and on task [21]. But in transportation tasks, we can schedule in anticipation of the difficulties that will occur in certain regions of the trip. We have a limited resource of attention, and we are trying to estimate and adjust this limited resource, in the face of both known and unknown difficulties.

Attention on transportation tasks may be directly driven by the environment -the physical situation drives the need for attention. A planning method might want to consider several factors related to the environment - how capable the robot is at navigating certain complex regions, the amount of trust in the robot's capability, and the communication connectivity to the robot throughout the route. These might determine an interaction scheme, leading to the assignment of supervisors and operators.

We don't want to oversimplify; the amount of attention to be allocated toward a problem is not totally determined by the environment; it is also driven by the quality of the user interface, as well as the capability of the human. In particular, schemes involving context switching will need to consider the amount of time it will take operators to gain situational awareness prior to taking control of a vehicle.

If our machines are running well, we might be able to monitor many with a single person. But if things go wrong, we need a capacity of human attention sufficient to both perform the intervention and continue the monitoring - we need to draw from a pool.

\section{Fan-Out}

The previous section suggests that the control of multiple robots on transportation tasks is complex, and leads us back to one of our motivating questions - how many robots a human can really control.

Olsen and Wood describe fan-out as a function of activity time (the time a robot moves) and interaction time (the amount of time a human may spend operating the robot). [20]. Fan out indicates how many robots are controllable at one time.

In the situations Olsen and Wood studied, the robots could all be deployed simultaneously; the staggering of a convoy was not part of their examples. In our case, we are measuring the time to get a set of robots from one place to another. We are bounded by two lines, as in figure 13 .

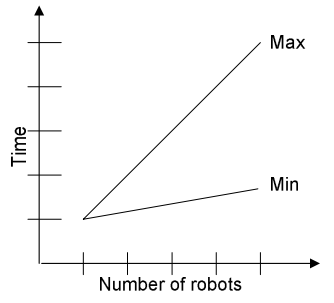

Figure 13. The range of time as robots are added.

The vertical time unit is the length of time $\mathrm{T}$ to traverse the route with one robot. For $N$ robots, we will spend at most $N^{*} T$ time - we can always stagger the start of the next robot's trip at the full route distance to avoid any attention problems. One might expect the minimum time to stay constant, but it may move up slightly, as in some situations every additional robot will need to be staggered by at least the length of the largest special zone in the route.

What then is the fan-out for a particular situation? We can deploy an infinite number of robots - but if we send the robots through one at a time, our effective fan-out is 1 , as we will achieve no parallelism. We need to normalize the number of robots involved by the elapsed time for all robots to move through a route. We define effective fanout for a particular route as:

$$
\text { EffectiveFanOut }=\frac{\mid \text { robots } \mid * \text { SingleRobotTraversalTime }}{\text { MultiRobotTraversalTime }}
$$

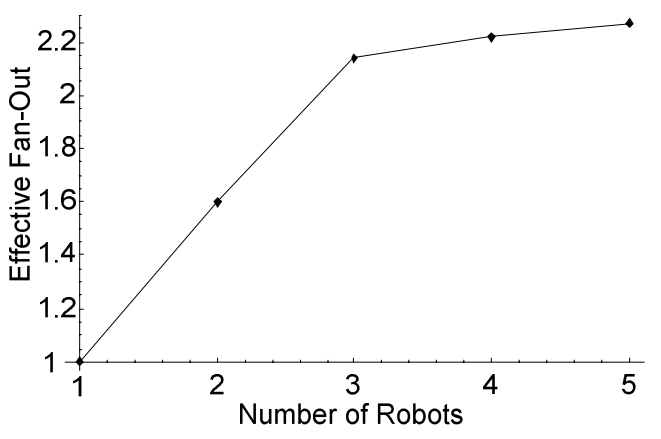

Figure 14. Effective Fan-Out for a simulation in which robots traverse the same path to the same destination, avoiding overlap in special regions. 
We multiply the number of robots by the amount of time it takes a single robot to move through the course, to establish the least efficient plan. We then divide by the fastest multiple robot route we can find for the given regions of the route.

Figure 14 shows how effective fan-out plateaus as the number of robots increases, on a simulation run in which all robots traverse a route where $30 \%$ of the route includes special regions (each point in the graph represents a mean value over 35 random routes). In other simulation runs with $70 \%$ special regions, we saw the effective fan-out fall as the number of robots increased beyond a certain point; this makes sense, as once the route is saturated by robots, the next additional robot may need to wait for the others to clear the route, effectively bringing the effective fan-out back down toward 1 .

Now let us consider the formulas of Olsen and Wood:

$$
\text { FanOut }=\frac{\text { ActivityTime }}{\text { InteractionTime }} \text {, }
$$

with ActivityTime = OverlapRatio * InterationTime + NeglectTime. The overlap ratio for teleoperation is 1 , as it requires full attention. In our first set of situations, we imagined interaction time only in special regions, so InteractionTime $=$ TimeInRegions. In these situations, Neglect time $=$ RouteTime - TimeInRegions. So, through algebraic simplification,

$$
\text { FanOut }=\frac{\text { RouteTime }}{\text { TimeInRegions }} .
$$

Sensibly, as the TimeInRegions increases, FanOut decreases.

Table 1. Effective Fan-Out prediction and simulation

\begin{tabular}{|l|l|l|l|}
\hline \%o of Special Regions & $\mathbf{3 0}$ & $\mathbf{5 0}$ & $\mathbf{7 0}$ \\
\hline Fan-Out Prediction & 3.3 & 2 & 1.4 \\
\hline EFO - Many routes & 2.8 & 1.7 & 1.2 \\
\hline EFO - One route & 2.3 & 1.4 & 1.1 \\
\hline
\end{tabular}

Table 1 shows the fan-out calculated with this formula, alongside with the mean values from two sets of simulations (35 trials on random routes were run for each cell of the table). In the top set, the cars are sent on different random routes. in the bottom set they are set on the same route. The measure predicts within about $20 \%$ the multi-route effective fan-out. The reader will notice that multiple routes have higher effective fan-out than single routes. An intuition as to why this happens can be gained by looking at diagrams $5 \mathrm{~d}$ and 6 ; multiple routes provide different shapes that, probabilistically, are more likely to fit together into a shorter route.

It is remarkable that the Olsen and Wood equation appears to be usable as a heuristic to gauge effective fanout. The formula can be easily computed, and needs no information on the actual locations of special regions only the ratio of these regions to the overall trip length.

Gauging effective fan-out might be used as a kind of feasibility test before proceeding to detailed planning. It would just be a first step; for example, in order to achieve an effective fan-out of 2, 4 robots may need to be staggered in a particular way to fit the particular route constraints.

\section{Conclusions}

We have shown that, if we need to teleoperate in special regions, then we can compute the shortest possible staggering of vehicles. We showed the computation for the multi-route situation is NP-complete.

Our main restriction is not computational, as we can still find routes for small numbers of robots. Our problem is attentional. Even if we can handle many vehicles at the same time, our need to spread our attention may result in a low effective fan-out.

This paper has offered several ideas towards supervisory and operator decision aids. The first is that if we can, ahead of time, designate special regions in which teleoperation will be necessary, then we might plan routes which will have at most one vehicle in a special region at a time.

Regions might be special because they are hard to teleoperate in. They might be special for a different reason - communication might be lost in regions defined by physical containment, such as tunnels. We might use a concept of communication distance to plan for such occasions - and to handle situations where communication is lost for unknown reasons. Utilizing a decision aid, we might use other autonomous vehicles to reestablish communication or intercept a malfunctioning vehicle.

Also, this concept of communication distance leads us to a way of thinking about the attention of operators. The more subsumed an operator is in an intervention, the longer the distance (the time to refocus attention) to the other vehicles becomes. This is neglect time - but neglect time that can be analyzed, and reduced through the proper allocation of attention.

We may consider using pools of operators the same way similar pools are used in call centers to handle bursts of activity. In conditions of extreme emergency, we may not want to shift autonomy back to machines; instead, we may want the option of focusing large amounts of human attention on the problem.

\section{Acknowledgements}

The authors wish to acknowledge the support by the National Science Foundation under grant numbers. 
0326309 and 0325123 . In addition, the authors would like to thank the anonymous reviewers for their suggestions.

\section{References}

[1] K. S. Barber and C. E. Martin, "Agent Autonomy: Specification, Measurement, and Dynamic Adjustment," Autonomy Control Software Workshop, Seattle, Washington, 1999.

[2] C. Castelfranchi, "Guarantees for Autonomy in Cognitive Agent Architecture," Intelligent Agents: ECAI-94 Workshop on Agents Theories, Architectures, and Languages, Berlin, 1994.

[3] R. Falcone and C. Castelfranchi, "Levels of Delegation and Levels of Adoption as the basis for Adjustable Autonomy.," in Lecture Notes in Artificial Intelligence, 2000, pp. 285-296.

[4] G. A. Dorais, R. P. Bonasso, D. Kortenkamp, B. Pell, and D. Schreckenghost, "Adjustable Autonomy for HumanCentered Autonomous Systems on Mars," First International Conference of the Mars Society, 1998.

[5] R. Falcone and C. Castelfranchi, "The human in the loop of a delegated agent: The theory of adjustable social autonomy," IEEE Transactions on Systems, Man, and Cybernetics Part A:Systems and Humans., vol. 31, no. 5, pp. 406-418, 2001.

[6] H. Hexmoor, "Case Studies of Autonomy," Thirteenth International Florida Artificial Intelligence Research Symposium Conference, Orlando, Florida, 2000.

[7] R. Parasuraman, T. B. Sheridan, and C. D. Wickens, "A Model for Types and Levels of Human Interaction with Automation," IEEE Transactions on Systems, Man, and Cyberntics - Part A: Systems and Humans, vol. 30, no. 3, pp. 286-297, 2000.

[8] T. B. Sheridan, Telerobotics, automation, and human supervisory control. Cambridge, Mass.: MIT Press, 1992.

[9] M. Lewis, K. Sycara, and I. Nourbakhsh, "Developing a Testbed for Studying Human-robot Interaction in Urban Search and Rescue," Proceedings of the 10th International Conference on Human Computer Interaction, 2003.

[10] J. L. Marble, D. J. Bruemmer, D. A. Few, and D. D. Dudenhoeffer, "Evaluation of Supervisory vs. Peer-Peer Interaction with Human-Robot Teams," Proceedings of the 37th Hawaii International Conference on System Sciences, 2004.

[11] S. Hughes, J. Manojlovich, M. Lewis, and J. Gennari, "Camera Control and Decoupled Motion for Teleoperation," Proceedings of the 2003 IEEE International Conference on Systems, Man, and Cybernetics, 2003.

[12] J. Wang, M. Lewis, and J. Gennari, "Interactive Simulation of the NIST USAR Arenas.," Proceedings of the 2003 IEEE International Conference on Systems, Man, and Cybernetics, 2003.
[13] J. Scholtz, B. Antonishek, and J. Young, "Evaluation of Operator Interventions in Autonomous Off-road Driving," PerMIS, 2003.

[14] J. Scholtz and S. Bahrami, "Human-Robot Interaction: Development of an evaluation methodology for the Bystander Role," to be presented at 2003 IEEE International Conference on Systems, Man \& Cybernetics., 2003.

[15] K. Sycara and M. Lewis, "Experiments in Implicit Control," Notes of the IJCAI 2003 Workshop on MixedInitiative Intelligent Systems, 2003.

[16] H. Hexmoor and T. Heng, "Air Traffic Control Agents: Landing and Collision Avoidance," International Conference in Artificial Intelligence, H. R. Arabnia, (ed), IC-AI, 2000, Las Vegas, 2000.

[17] H. Hexmoor, "Stages of Autonomy Determination," IEEE Transactions on Man, Machine, and cyberneticsPart C (SMC-C), vol. 31, no. 4, pp. 509-517, 2002.

[18] J. V. Nickerson and R. Reilly, "A Model for Investigating the Effects of Machine Autonomy on Human Behavior," Proceedings of the 37th Hawai'i International Conference on System Sciences, 2004.

[19] J. Scholtz, B. Antonishek, and J. Young, "Evaluation of a Human-Robot Interface: Development of a Situational Awareness Methodology," Proceedings of the 37th Hawaii International Conference on System Sciences, 2004.

[20] D. R. Olsen and S. B. Wood, "Fan-out: measuring human control of multiple robots," Conference on Human factors in computing systems, 2004.

[21] J. W. Crandall and M. A. Goodrich, "Characterizing efficiency of human robot interaction: a case study of shared-control teleoperation," IEEE/RSJ International Conference on Intelligent Robots and Systems, 2002.

[22] D. R. Olsen and M. A. Goodrich, "Metrics for Evaluating Human-Robot Interaction," Permis, 2003.

[23] M. R. Garey and D. S. Johnson, Computers and intractability: a guide to the theory of NP-completeness. San Francisco: W. H. Freeman, 1979.

[24] J. V. Nickerson, "A Concept of Communication Distance and its Application to Six Situations in Mobile Environments," IEEE Transactions on Mobile Computing, vol. 4, no. 1, 2005.

[25] T. Kamakaris and J. V. Nickerson, "Connectivity maps: Measurements and applications," Hawaii International Conference on System Sciences, 2005.

[26] L. Dabbish and R. Kraut, "Controlling Interruptions: Awareness Displays and Social Motivation for Coordination," CSCW, 2004.

[27] D. S. McCrickard, R. Catrambone, C. M. Chewar, and J. T. Stasko, "Establishing tradeoffs that leverage attention for utility: empirically evaluating information display in notification systems," Int. J. Human-Computer Studies, vol. 58, pp. 547-582, 2003.

[28] T. Fong and C. Thorpe, "Vehicle Teleoperation Interfaces," Autonomous Robots, vol. 11, no. 1, pp. 0918,2001 